\title{
Switzerland and the European Union
}

Citation for published version (APA):

Günthardt, J. A. (2020). Switzerland and the European Union: the implications of the institutional framework and the right of free movement for the mutual recognition of professional qualifications. [Doctoral Thesis, Maastricht University]. Maastricht University. https://doi.org/10.26481/dis.20201217jg

\section{Document status and date:}

Published: 01/01/2020

DOI:

10.26481/dis.20201217jg

Document Version:

Publisher's PDF, also known as Version of record

\section{Please check the document version of this publication:}

- A submitted manuscript is the version of the article upon submission and before peer-review. There can be important differences between the submitted version and the official published version of record.

People interested in the research are advised to contact the author for the final version of the publication, or visit the DOI to the publisher's website.

- The final author version and the galley proof are versions of the publication after peer review.

- The final published version features the final layout of the paper including the volume, issue and page numbers.

Link to publication

\footnotetext{
General rights Owners
rights.

- You may freely distribute the URL identifying the publication in the public portal. please follow below link for the End User Agreement:

www.umlib.nl/taverne-license

Take down policy

If you believe that this document breaches copyright please contact us at:

repository@maastrichtuniversity.nl

providing details and we will investigate your claim.
}

Copyright and moral rights for the publications made accessible in the public portal are retained by the authors and/or other copyright owners and it is a condition of accessing publications that users recognise and abide by the legal requirements associated with these

- Users may download and print one copy of any publication from the public portal for the purpose of private study or research.

- You may not further distribute the material or use it for any profit-making activity or commercial gain

If the publication is distributed under the terms of Article $25 \mathrm{fa}$ of the Dutch Copyright Act, indicated by the "Taverne" license above, 


\section{Summary, conclusions and recommendations}

\section{$9.1 \quad$ Summary}

This research analysed the acquis suisse for the mutual recognition of professional qualifications, or in other words the recognition of diplomas between Switzerland and the EU, looking particularly at selected health and legal professions. This research not only examines the applicable secondary law, namely the Professional Qualifications Directive, but also examined whether primary law applies in this context between Switzerland and the EU. For this purpose, the institutional mechanism which is inherently linked to this topic was discussed because future relations between Switzerland and the EU, as reflected in the Draft Institutional Framework Agreement, could have a large impact on the interpretation of the acquis suisse.

\subsubsection{The institutional framework between Switzerland and the EU (Part I)}

This study began with Part I looking at the institutional mechanism between Switzerland, the EU and its Member States, and how this mechanism will be altered in the future. It shows that challenges, such as the popular initiative against mass immigration, have not hindered the so-called bilateral path between Switzerland and the EU. It also considers the current institutional challenges in the search for a common body of interpretation for the bilateral agreements.

The Draft Institutional Framework Agreement was under public consultation in spring 2019. It would create an arbitration body where a 'referral' to the CJEU would be permissible for certain market access agreements. Considering the extent of the foreseen 'reference procedure', the Draft Institutional Framework Agreement indicates that a very moderate level of guidance would be requested from the CJEU for most bilateral market access agreements, and possibly lead to being in conformity with the autonomy of the EU legal order. In a comparison with similar dispute settlement mechanisms, it was briefly discussed whether the arbitration body has been left any discretion not to refer a case to the CJEU, which can be answered in the affirmative (with the application of the acte clair doctrine).

The Draft Institutional Framework Agreement is a compromise between the EU and Switzerland. It seems that the compromise may prove difficult to accept for many Swiss 
people, as it crosses some so-called red lines, such as the eight-day waiting period for service providers (without the classic divide between right and left-wing politicians). In addition to the crossing of red lines, there is also opposition to the competence of the CJEU, as it is considered by some as a court of the 'opposite side', and which implies that the CJEU is not impartial.

Importantly, the interpretation of association agreements and in particular of the Agreement on the Free Movement of Persons ('AFMP') was explored. While the CJEU's interpretation of the Ankara Agreement is similar to the progressive case law of the internal market in numerous cases, it was shown that it explicitly returned to the Polydor principle for the interpretation of the Ankara Agreement in the case Demirkan and for the AFMP in the case Grimme. It was even once doubted by an Advocate General whether primary law forms part of the AFMP or only the listed secondary legislation in the Annexes of the AFMP in the case Ettwein. It was also explained that according to scholars this return to the Polydor case law could have been caused by the restrictive judgments of some national courts. It was therefore noted that, in particular, recent criminal law judgments of the Swiss Federal Court could lead to a more extensive return of the Polydor case law under the AFMP because the interpretation of the CJEU depends largely on the context of the respective international agreements. Despite the return of the Polydor case law, it was also indicated that the Swiss Federal Court follows the case law of the CJEU, even if it is later altered. The CJEU has never openly followed the jurisprudence of the Swiss Federal Court (with one exception). It was shown that the type of judicial dialogue that exists between the EFTA Court and the CJEU does not take place between the Swiss Federal Court and the CJEU. The judicial dialogue is explicitly mentioned in the Draft Institutional Framework Agreement between Switzerland and the EU. It has been explained that the EFTA Court has even gone beyond judicial dialogue and introduced a reversed Polydor principle aiming for the same result as in the internal market, regardless of the CJEU's interpretation.

Finally, it is clear that a rule of interpretation can only be applied for concepts which are part of the AFMP. But how to determine the concepts that have been adopted by the acquis suisse has not been solved. The complex structure of the AFMP and the combination of primary and secondary law make finding out which concepts of EU law are also part of the AFMP a daunting task. This issue will remain unsolved by the Swiss-EU Draft Institutional Framework Agreement of 23 November 2018. 


\subsubsection{Free movement of persons between Switzerland and the EU (Part II)}

Part II discussed the fundamental freedoms which have partly developed under the acquis suisse. While direct and indirect discrimination are prohibited (if they are not justified) for all applicable fundamental freedoms (freedom of workers, freedom of establishment and freedom of services) and have horizontal effect (left unanswered in some recent cases of the Swiss Federal Court), it is still disputed whether free movement restrictions are covered by the AFMP. There is currently no case law where the CJEU has expressly stated that not only discrimination but also free movement restrictions are covered by the AFMP (or any other association agreement). ${ }^{2420}$ The Swiss Federal Court did not take an open position but only ruled implicitly in favour of the recognition of professional qualifications based on primary law, or rather a pragmatic approach (possibly based on the principle of proportionality).

While in the recent Grand Chamber judgment Picart the CJEU interpreted the AFMP by its wording and held that a self-employed person 'must pursue his self-employed activity in the territory of a Contracting Party other than which he is a national', it held in another Grand Chamber judgment, Wächtler, that 'restrictions' on the free movement provisions from the State of origin are prohibited. For the recognition of professional qualifications, the Swiss courts have almost consistently ruled that the cross-border situation is deemed 'sufficient'.

It was also pointed out that non-economically active persons (e.g. students) are most likely excluded from the scope of the AFMP for access to university education due the wording of Article 24(4) of Annex I to the AFMP. Even if the case law suggests that persons who are not economically active may also not invoke Article 2 AFMP in this situation (similar to Article 18 TFEU as a form of subsidiary protection and contrary to the case law of lower Swiss courts), economically active students may in principle invoke the fundamental freedoms, such as the free movement of workers in conjunction with Article 2 AFMP.

\subsubsection{Mutual recognition of professional qualifications (Part III)}

In Part III it became clear how the mutual recognition of professional qualifications and its fundamental principles were developed. The evolution of the principles of mutual trust

2420 The term 'restriction' has been used by the CJEU in this context: e.g. Case C-581/17, Martin Wächtler v Finanzamt Konstanz, ECLI:EU:C:2019:138, para. 67. Even if the CJEU explicitly used the term 'restrictions' in Wächtler, the same wording as in the internal market does not mean that the term bears the same meaning after everything that has been discussed about the Polydor principle in this study. 
and country of origin is based on the case law of the fundamental freedoms. It was again emphasised, with regard to the respective case law, that the Swiss Federal Court in particular implicitly acknowledges the concept of recognition of professional qualifications based on a pragmatic approach (possibly on the principle of proportionality), while the Swiss government even openly acknowledges the regime of recognition of professional qualifications based on the Hocsman and Morgenbesser case law. The discussion of the electrician's profession also showed that the FAC seems to accept this concept because partial recognition under secondary law is not yet part of the acquis suisse. It was demonstrated that, from a dogmatic standpoint, the recognition of professional qualifications could either be based on primary law or on a pragmatic approach (similar to the reversed Polydor principle of the EFTA Court) but not solely on the principle of proportionality. This would have an impact for the recognition of third country diplomas as well as partial recognition based on primary law. It contradicts earlier and lesser known case law of the FAC which refused to apply the case law of Vlassopoulou and Dressen as a subsidiary layer of protection when secondary law does not apply.

In this context, it was also explored for third country diplomas that the case law of Tennah-Durez which states that Member States must also recognise diplomas even if the training was not mainly obtained in the EU, as long as the diploma is awarded by an authority of an EU Member State, still applies for the sectoral system of recognition according to the European Commission.

With the withdrawal of the UK from the EU, the protection of acquired rights was discussed, as explicitly foreseen in Article 23 AFMP. Some of the legal uncertainties have now been clarified with a new Swiss-UK Brexit Agreement that apply on the withdrawal of the UK. This agreement protects acquired rights, rights in the process of being acquired and even certain persons who have not obtained a diploma. Further, the recognition of third country diplomas is regulated. Recognitions based on primary law and the auditor's profession are not mentioned in the agreement.

\subsubsection{Mutual recognition of professional qualifications for selected health and legal professions (Part IV)}

In Part IV, the relevant case law of the CJEU, the EFTA Court and Swiss courts was discussed for selected health and legal professions. For the pursuit of the profession of a medical doctor in the host Member State, an interesting decision of the EFTA Court was $-422-$ 
explained, allowing the host Member State to refuse recognition if the migrant does not have the necessary linguistic knowledge. In the present author's opinion, it is however more convincing to adopt a two-stage approach which is in conformity with the case law of the CJEU. By application of the two-stage approach, the recognition of professional qualifications is in principle automatic, while the exercise of the profession can be prohibited due to a lack of linguistic knowledge.

Swiss courts have consistently held that diplomas awarded by private institutions are considered to be part of public law if the relevant institutions are accredited as required by Swiss law. This also applies to specialised doctors of medicine. In an important decision with five judges, in 2017, the FAC ruled that even a private diploma showing a specialisation in endocrinology must be recognised if required by federal law based on the general system. It was revealed that the FAC did not discuss in this case whether the Angerer case law must be followed for a medical specialty not listed in Annex V of the Professional Qualifications Directive under the acquis suisse, despite the fact that Article 10 of the Professional Qualifications Directive only applies for specific and exceptional reasons. This can be answered in the affirmative. The application of this case law would therefore have led to the same result. It was also held obiter dictum referring to the decision of the CJEU in Bobadilla that the recognition principles would even apply for the non-regulated professions but without giving a reasoning. In the end, the decision was recently annulled by the Swiss Federal Court on grounds of a lack of legal basis of the requirement in June 2019.

In another important leading case of the FAC of 2018, a measure which stipulates that doctors must not be approved by health insurance funds unless they show that they have three years of practical experience in Switzerland was found to be indirectly discriminatory by the FAC, but justified. Division III of the FAC held that the meaning of Article 55 of the Directive ${ }^{2421}$ was not sufficiently clear to be applied in case of mere restrictions and in the light of the Swiss Federal Court case law despite the fact that the measure was found to be indirectly discriminatory. The standstill clause of the AFMP was not assessed despite the fact that the measure was found to be indirectly discriminatory as opposed to the cited leading

2421 'Without prejudice to Article 5(1) and Article 6, first subparagraph, point (b), Member States which require persons who acquired their professional qualifications in their territory to complete a preparatory period of in-service training and/or a period of professional experience in order to be approved by a health insurance fund, shall waive this obligation for the holders of evidence of professional qualifications of doctor and dental practitioner acquired in other Member States'. 
case of the Swiss Federal Court. It was never answered whether violations of the standstill clause in the AFMP can be justified per analogiam to case law of the CJEU for the standstill clause under the Ankara Agreement.

Concerning the health professions, the new provision for pharmacists which requires a specialised diploma for independent practise was assessed. From the case law of the CJEU it can be seen that the recognition of specialised pharmacist diplomas falls under the general system of recognition. Even if it is argued that the diploma for 'specialised pharmacist' does not fall under the scope of the Professional Qualifications Directive due to the fact that only the exercise is restricted, or if it is argued that the measure is in conformity with the Directive, the measure qualifies either as indirect discrimination or more likely as a free movement restriction which must be justified if free movement restrictions are covered by the AFMP or a pragmatic approach is applied. This view is also supported by the relevant case law of the EFTA Court.

While the implementation of the lawyer's and the patent attorney's professions in Switzerland was shown to be largely unproblematic with the exception of a cantonal judgment that erroneously exempts family members from the scope of the Facilitating Practice Directive, the legal situation for the notarial profession is unsatisfactory, as the courts did not clarify whether they can benefit from free movement under the acquis suisse.

Since at least primary law applies for the notarial profession but according to the Swiss Competition Commission even secondary law applies based on the national implementation of Title II of the Professional Qualifications Directive and the application of the Qualifications Directive before its revision, free movement should in principle be allowed for notaries under the acquis suisse. For the notarial profession, it was explored that the public authority exception to the AFMP must be interpreted according to the case law of the CJEU. There is no good reason to disapply the case law of the CJEU concerning notaries despite a slightly nuanced wording of the public authority exception due to the rule of homogeneity foreseen in Article 16(2) AFMP. This means that notaries generally do not fall under this exception. It was also highlighted that Swiss nationals and nationals from EU Member States may rely on the AFMP against their State of origin even if a profession falls under the public authority exception according to the Brouillard decision of the CJEU.

In addition, it was noted that the FAC has not clarified whether auditors fall under secondary or primary law under the acquis suisse at all. It was claimed that it is unconvincing 
that they cannot profit from free movement because this profession is not mentioned under the exemptions of the AFMP.

Finally, the last part of this study showed the situation concerning free movement of legal trainees within the meaning of the Morgenbesser (and Peśla) case law, and that it is part of the acquis suisse pursuant to the statement of the Swiss Government, and that the Swiss authorities must apply a holistic view when assessing the free movement of legal trainees. This view is also supported by recent case law of the Swiss Federal Court. On 31 January 2020, the Swiss Federal Court upheld a cantonal decision that applied the lessons of the Peśla judgment but which did not grant access to legal traineeship in the specific case. 


\section{Samenvatting}

Deze studie beschrijft en analyseert het acquis suisse met betrekking tot de wederzijdse erkenning van beroepskwalificaties, met andere woorden de erkenning van diploma's, tussen Zwitserland en de EU. Daarbij wordt de focus gelegd op bepaalde beroepen in de gezondheidszorg en in de juridische beroepen. In deze studie wordt daarnaast niet alleen het toepasselijke secundaire recht geanalyseerd, zoals met name Richtlijn 2005/36/EG ("Beroepserkenningsrichtlijn"), maar ook de vraag onderzocht of de beginselen inzake wederzijdse erkenning zoals deze zijn gelezen in het primaire EU recht ook van toepassing zijn in de rechtsrelatie tussen Zwitserland en de EU. Daartoe wordt het institutionele kader, dat nauw verband houdt met dit onderwerp, en de ontwikkelingen in de betrekkingen tussen Zwitserland en de EU, en met name de kaderovereenkomst, uiteengezet omdat deze een belangrijke invloed kunnen hebben op de toekomstige interpretatie van het acquis.

\subsection{Institutioneel kader tussen Zwitserland en de EU (deel I)}

Deel I van deze studie gaat over het institutionele kader dat de relaties tussen Zwitserland en de EU beheerst, de geschiedenis ervan en de mogelijke wijzigingen in de toekomst. Dit gedeelte toont aan dat de verschillende struikelblokken, zoals het initiatief voor massale immigratie (Masseneinwanderungsinitiative), de ontwikkeling van de zogenaamde 'bilaterale weg' tussen Zwitserland en de EU niet hebben tegengehouden. Daarnaast werden ook de huidige institutionele uitdagingen met betrekking tot het vinden van een geschikte (rechterlijke) instantie voor de uniforme interpretatie van de bilaterale overeenkomsten geanalyseerd.

In het voorjaar van 2019 werd over de ontwerp-kaderovereenkomst tussen Zwitserland en de EU een openbare raadpleging gehouden. Deze kaderovereenkomst zou een scheidsgerecht in het leven roepen met de mogelijkheid om prejudiciële vragen te stellen aan het Hof van Justitie van de Europese Unie (HvJ-EU) voor bepaalde markttoegangsovereenkomsten. Gezien de wijze van inkadering van de deze verwijzingsprocedure in de ontwerp-kaderovereenkomst wordt duidelijk dat de invloed van het Hof van Justitie op de interpretatie van deze markttoegangsovereenkomsten relatief bescheiden zou zijn en tegelijkertijd de vorm van geschillenbeslechting mogelijk ook in overeenstemming met de autonomie van het recht van de Unie zou zijn. Er wordt daarnaast 
Chapter 10

kort geanalyseerd of het scheidsgerecht een discretionaire bevoegdheid heeft om vragen aan het Hof van Justitie van de Europese Unie voor te leggen, hetgeen kan worden bevestigd op basis van vergelijking met andere mechanismen voor geschillenbeslechting, het (toepassing van de zogenaamde acte clair-doctrine).

De ontwerp-kaderovereenkomst is een compromis tussen Zwitserland en de Europese Unie. Niettemin zal deze mogelijk voor veel Zwitsers moeilijk te aanvaarden zal zijn omdat het bepaalde zogenaamde 'rode lijnen' overschrijdt, zoals met betrekking tot de 8-dagenregel voor dienstverleners (een kritiek die zowel van links als rechts klinkt). Naast het overschrijden van de rode lijnen is er het bezwaar dat het Hof van Justitie een rechterlijke instantie is van de EU, voor sommigen dus een gerecht van 'de andere kant', waarmee geïmpliceerd wordt dat het Hof niet onpartijdig zou oordelen.

Daarnaast is de interpretatie van internationale verdragen en met name de interpretatie van de Overeenkomst over het Vrije Verkeer van Personen tussen Zwitserland en de EU (OVVP) onderzocht. Hoewel het Hof van Justitie van de Europese Union (HvJ-EU) in veel gevallen een progressieve interpretatie toepast en interne marktbeginselen heeft geïmporteerd uit het EU-recht in zowel de rechtsrelatie EU-Zwitserland, maar ook in het kader van de Associatieovereenkomst EU-Turkije (de 'Overeenkomst van Ankara') heeft het in recente rechtspraak, zoals Demirkan onder de Overeenkomst van Ankara en in de zaak Grimme onder de OVVP, teruggegrepen naar het Polydor-principe. De advocaat-generaal in de zaak Ettwein vroeg zich zelfs af of het primaire EU-recht deel überhaupt uitmaakt van de OVVP of dat alleen het uitdrukkelijk genoemde secundaire recht deel uitmaakt van het acquis suisse. In dit deel komen daarnaast de opvattingen van enkele auteurs aan bod die de terugkeer naar het Polydor-principewijten is aan een aantal restrictieve uitspraken van nationale rechtbanken. Indien juist, is het goed denkbaar dat het Polydor-principe in toenemende mate zal worden toegepast, in het licht van een aantal recente restrictieve uitspraken van de afdeling strafrecht van het Zwitserse Federale Hof (het Bundesgericht), nu de interpretatie van het Hof van Justitie nu eenmaal in grote mate afhangt van de context van de desbetreffende overeenkomst.

Echter, ondanks de terugkeer naar het Polydor-principe toont een analyse van de rechtspraak aan dat het Bundesgericht het Hof van Justitie volgt, ook als deze zijn jurisprudentie later heeft gewijzigd. Het Hof van Justitie heeft daarentegen nooit expliciet het Bundesgericht gevolgd, op één uitzondering na. Ook is gebleken dat de samenwerking tussen de rechtbanken of de justitiële dialoog, zoals die tussen het Hof van Justitie en het EVA-Hof bestaat, niet plaatsvindt tussen het Bundesgericht en het HvJ-EU. De justitiële dialoog is - 438 - 
echter wel expliciet opgenomen in de kaderovereenkomst. Er is daarnaast uiteengezet dat het EVA-Hof zelfs verder gaat dan slechts een justitieel dialoog en een omgekeerd Polydorprincipe heeft ontwikkeld. Deze komt op neer komt dat het de EER-overeenkomst (ongeacht van de precieze interpretatie van het Hof van Justitie) op een wijze interpreteert opdat hetzelfde resultaat wordt bereikt als in het EU interne marktrecht.

Het is duidelijk dat de door het Hof van Justitie ontwikkelde interpretatieregels alleen van toepassing kunnen zijn op de begrippen die daadwerkelijk deel uitmaken van de OVVP. Dit betekent dat nog geen oplossing is gevonden voor de interpretatie van de begrippen die deel uitmaken van het acquis suisse. De complexe structuur van de OVVP en de combinatie van primaire en secundaire recht maken het niet eenvoudig om te bepalen welke EU-rechtelijke concepten ook onderdeel zijn van de OVVP. De EU-Zwitserland kaderovereenkomst van 23 november 2018 verhelpt dit probleem ook niet.

\subsection{Vrij verkeer van personen tussen Zwitserland en de EU (deel II)}

In deel II worden de fundamentele vrijheden besproken, die slechts gedeeltelijk in het acquis suisse zijn ontwikkeld. Hoewel zowel geaccepteerd is dat zowel directe als indirecte discriminatie (tenzij gerechtvaardigd) verboden zijn voor alle toepasselijke fundamentele vrijheden (werknemers, vestiging en diensten), en deze vrij verkeersrechten rechtstreekse horizontale werking hebben (overigens recent door het Bundesgericht opengelaten), wordt nog steeds betwist of de OVVP ook een verbod op beperkingen van vrij verkeersrechten omvat. Uit de analyse blijkt dat er geen enkel oordeel van het Hof van Justitie is dat expliciet een verbod op beperkingen voor de OVVP (of voor een andere associatieovereenkomst) bevestigt. ${ }^{2429}$ Het Bundesgericht heeft niet openlijk, maar wel impliciet steun uitgesproken voor zo een interpretatie waar het erkenning van diploma's op basis van het primaire recht betreft. Dit uitspraak betreft echter geen principieel maar eerder een pragmatisch standpunt (mogelijk ingegeven door het proportionaliteitsbeginsel).

Terwijl het Hof in het recente arrest Picart (Grote kamer) de OVVP restrictief uitlegt op basis van de bewoordingen, namelijk dat een onderdaan van Zwitserland of de EU als

2429 De term "beperking" is door het Hof van Justitie gebruikt: zie zaak C-581/17, Martin Wächtler/Finanzamt Konstanz, ECLI:EU:C:2019:138, paragraaf 67. Hoewel het Hof van Justitie de term "beperking" heeft gebruikt, kan het gebruik van deze formulering - volgens wat in deze studie over het Polydor-principe is geschreven - niet automatisch leiden tot de conclusie dat de term dezelfde betekenis heeft als in het interne marktrecht. 
Chapter 10

zelfstandige op het grondgebied van de andere overeenkomstsluitende partij moet verblijven, oordeelde datzelfde Hof in de zaak Wächtler (Grote kamer) dat belemmeringen van het vrije verkeer van personen geïntroduceerd door de staat van herkomst verboden zijn. Wat de erkenning van diploma's betreft, hebben de Zwitserse rechtbanken in de grotendeels consistente jurisprudentie besloten dat een grensoverschrijdend element voldoende is voor de toepassing van de OVVP en de richtlijn inzake de erkenning van beroepskwalificaties.

In dit deel wordt ook vermeld dat personen die geen economische activiteit uitoefenen (bijvoorbeeld studenten) waarschijnlijk buiten het toepassingsgebied van de OVVP vallen als gevolg van de formulering van artikel 24, lid 4, van bijlage I bij de OVVP, waar het de toegang tot het hoger onderwijs betreft. Personen in deze situatie kunnen zich waarschijnlijk niet beroepen op artikel 2 van de OVVP (non-discriminatie naar analogie van artikel 18 VWEU, overigens een opvatting die door lagere rechters niet altijd wordt gedeeld). Economisch actieve studenten kunnen zich vanwege deze status echter in beginsel wel beroepen op de fundamentele vrijheden in verband met artikel 2 van de OVVP.

\subsection{Erkenning van beroepskwalificaties (erkenning van diploma's; deel}

\section{III)}

Deel III belicht het ontstaan van de basisprincipes van de wederzijdse erkenning van diploma's. De ontwikkeling van de beginselen van wederzijds vertrouwen en het 'land van herkomst'-beginsel is gebaseerd op de jurisprudentie met betrekking tot de fundamentele vrijheden. In dit deel wordt behalve de al eerdere genoemde pragmatische erkenning van het diploma's door het Bundesgericht (ingegeven door het evenredigheidsbeginsel) er ook aan herinnerd dat de Zwitserse federale overheid zich openlijk heeft uitgesproken voor de rechtsprincipes met betrekking tot de erkenning van diploma's zoals deze voortvloeit uit de jurisprudentie in de zaken Hocsman en Morgenbesser. Uit de bespreking van de jurisprudentie over het beroep van elektricien lijkt ook de Federale Administratieve Rechtbank deze aanpak te volgen, aangezien de secundaire wetgeving inzake gedeeltelijke erkenning momenteel nog geen deel uitmaakt van het acquis suisse. Daarnaast wordt beargumenteerd dat erkenning van diploma's óf op basis van een lezing van het primaire recht in de Zwitserse context gebaseerd kan worden of op een pragmatische aanpak (gestoeld op het omgekeerde Polydor-principe van het EVA-Hof), maar niet slechts op het evenredigheidsbeginsel. Deze opvatting heeft zowel gevolgen voor de erkenning van 
diploma's van derde landen en de gedeeltelijke erkenning vandiploma's en is in tegenspraak met eerdere en weinig bekende jurisprudentie van de Federale Administratieve Rechtbank, dat weigerde de jurisprudentie in Vlassopoulou en Dressen toe te passen als subsidiair regime waar de Richtlijn niet van toepassing was.

In dit verband werd ook de jurisprudentie in de zaak Tennah-Durez onderzocht, waarin het Hof van Justitie oordeelde dat de lidstaten in het kader van het sectorale stelsel voor diploma-erkenning ook diploma's moeten erkennen die niet gebaseerd zijn op een overwegend in de Unie gevolgde beroepsopleiding, zolang het diploma door een EU-lidstaat is afgegeven. De Commissie is van mening dat deze jurisprudentie ook relevant is in het kader van de huidige richtlijn inzake beroepskwalificaties.

Met het oog op het vertrek van het Verenigd Koninkrijk uit de EU, werden de bescherming van verworven rechten en aanspraken besproken zoals voorzien in Artikel 23 OVVP. Sommige juridische onduidelijkheden konden worden verduidelijkt door de ontwerpovereenkomst tussen Zwitserland en het Verenigd Koninkrijk over de rechten van burgers in geval van opheffing van de Overeenkomst over het Vrije Verkeer van Personen (OVVP). Deze overeenkomst beschermt verworven rechten met betrekking tot de erkenning van diploma's, evenals verworven rechten en zelfs bepaalde personen die nog geen diploma hebben. Ook de erkenning van diploma's van derde landen is geregeld. De overeenkomst bevat geen bepalingen over de erkenning op grond van het primaire recht. Ten slotte wordt in de overeenkomst het beroep van auditor (opleiding tot auditor, fiduciair of belastingdeskundige) niet erkend.

\subsection{Erkenning van beroepskwalificaties voor geselecteerde gezondheids- en juridische beroepen (deel IV)}

In dit vierde deel van het proefschrift werd de relevante jurisprudentie van Hof van Justitie van de Europese Unie (HvJ-EU), het EVA-Hof en de Zwiterse rechtbanken met betrekking tot geselecteerde gezondheidszorgberoepen en juridische beroepen geanalyseerd. Een interessant besluit van het EVA-Hof werd becommentarieerd met betrekking tot de beroepsuitoefening van een vrouwelijke arts, op grond waarvan de ontvangende lidstaat de erkenning van het diploma kan weigeren in geval van onvoldoende talenkennis. Naar de mening van auteur dezes zou het overtuigender zijn om te kiezen voor een tweestappenbenadering, die in overeenstemming is met de jurisprudentie van het Hof van 
Justitiet. Bij de tweestappenbenadering is de erkenning van diploma's in het kader van het sectorale stelsel automatisch, terwijl de beroepsuitoefening kan worden geweigerd op grond van onvoldoende talenkennis.

Zwitserse rechtbanken zijn er altijd van uitgegaan dat door particuliere instellingen afgegeven diploma's als publiekrechtelijk worden beschouwd indien de desbetreffende instellingen zijn geaccrediteerd in overeenstemming met de Zwitserse wet. Dit is ook van belang voor specialisaties van artsen. In een belangrijke uitspraak van de Federaal Administratieve Rechtbank uit 2017, geveld door een meervoudige kamer, oordeelde deze instantie dat de titels van een privaatrechtelijke voortgezette opleiding voor artsen ('voortgezette opleiding voor de specialisatie reproductieve geneeskunde en gynaecologische endocrinologie') ook onderworpen waren aan de regels inzake de erkenning van diploma's. De Federale Administratieve Rechtbank besprak echter niet of de beginselen uit de zaak Angerer diende te worden gevolgd voor een specialiteit niet opgenomen in bijlage V van de richtlijn betreffende de erkenning van beroepskwalificatie onder de acquis suisse, niettegenstaande het feit dat artikel 10 slechts kan worden toegepast in specifieke en uitzonderlijke situaties. In dit deel werd deze vraag bevestigend beantwoord. De toepassing van deze rechtspraak zou overigens tot het zelfde resultaat hebben geleid, namelijk de toepasselijkheid van het algemene stelsel. Obiter dictum verwees de rechtbank overigens nog naar de uitspraak van het Hof van Justitie in Bobadilla, en stelde dat de regels voor de erkenning van diploma's ook van toepassing zouden zijn op niet-gereglementeerde beroepen zonder dit echter nader te motiveren. Uiteindelijk is het besluit recent (juni 2019) in beroep vernietigd door de Bundesgericht wegens het ontbreken van een rechtsgrondslag voor dit vereiste

In een mijlpaalbeslissing van de Bundesgericht in 2018 werd een regeling die stelt dat artsen drie jaar praktijkervaring in Zwitserland nodig hebben voordat zij erkend mogen worden door zorgverzekeraars, geclassificeerd als indirect discriminerend door de Federaal Adminstratieve Rechtbank, maar gerechtvaardigd. De betreffende kamer oordeelde dat de formulering van artikel 55 van de richtlijn ${ }^{2430}$ betreffende de erkenning van

2430 'Onverminderd artikel 5, lid 1, en artikel 6, eerste alinea, onder b), verlenen de lidstaten die van degenen die op hun grondgebied hun beroepskwalificaties hebben verworven, eisen dat zij een voorbereidende stage volbrengen en/of een periode van beroepservaring doorlopen om bij een ziektekostenverzekering te kunnen worden gecontracteerd, vrijstelling van deze verplichting aan de houders van in een andere lidstaat verworven beroepskwalificaties van arts of beoefenaar van de tandheelkunde.' 
beroepskwalificaties niet duidelijk genoeg was om te worden toegepast in het licht van de jurisprudentie van het Bundesgericht inzake beperkingen, niettegenstaande het feit dat de rechter de maatregel indirect discriminerend achtte. De standstill-clausule van de OVVP werd niet onderzocht, hoewel er, in tegenstelling tot de situatie onderliggende de genoemde uitspraak van het Bundesgericht, sprake was van indirecte discriminatie. Het is onduidelijk of schendingen van de standstill-clausule kunnen worden gerechtvaardigd naar analogie van de jurisprudentie van het Hof van Justitie in het kader van de Overeenkomst van Ankara.

Met betrekking tot de beroepen in de gezondheidszorg is de nieuwe regeling voor apothekers kort besproken dat een gespecialiseerd diploma vereist voor zelfstandige beroepsbeoefening. Volgens de jurisprudentie van het Hof van Justitie valt een dergelijke specialisatie onder het algemene stelsel van diploma-erkenning. Ongeacht de vraag of dit systeem al dan niet in overeenstemming is met de bepalingen van de richtlijn betreffende de erkenning van beroepskwalificaties, gezien alleen de uitoefening van het beroep gereglementeerd is, vormt dit vereiste ofwel indirecte discriminatie ofwel een beperking van het recht op vrij verkeer die gerechtvaardigd moet worden (indien beperkingen onder de OVVP vallen). Dit wordt ondersteund door de relevante jurisprudentie van het EVA-Hof.

Hoewel de in dit deel verrichte analyse aantoont dat de Zwitserse implementatie met betrekking tot de beroepen van advocaat en octrooigemachtigde grotendeels onproblematisch is, met uitzondering van een uitspraak die weigerde familieleden van EU-burgers toe te staan zich op de vestigingsrichtlijn voor advocaten te beroepen, is de juridische situatie voor het beroep van notaris momenteel onbevredigend, aangezien de rechtbanken op dit punt nog geen duidelijkheid hebben kunnen verschaffe of deze beroepsgroep zich op vrij verkeer kan beroepen in de context van de acquis suisse.

Volgens de mededingingscommissie is het secundaire recht op grond van het acquis suisse - door de omzetting van titel II van de richtlijn inzake de erkenning van beroepskwalificaties in nationaal recht en de toepassing van de richtlijn vóór de herziening ervan - nog steeds van toepassing op notarissen. Het staat daarnaast vast dat het primaire recht in ieder geval subsidiair van toepassing is op notarissen. Bijgevolg moet het vrije verkeer van notarissen ook in het kader van het acquis suisse worden toegepast. Voor het notariaat werd ook vastgesteld dat de uitzondering met betrekking tot de uitoefening van het openbaar gezag moet worden begrepen in het licht van de jurisprudentie van het Hof van Justitie. Er geen goede reden om op basis van kleine taalkundige verschillen een van het interne marktrecht afwijkende interpretatie van de deze uitzondering gezien het 
concordantiebeginsel vervat in artikel 16, lid 2, OVVP. Dit betekent dat de activiteiten van notarissen niet onder de uitzondering 'bekleed met openbaar bezag' vallen. Er werd ook op gewezen dat Zwitserse onderdanen en onderdanen van EU-lidstaten - onder verwijzing naar het oordeel van het Hof van Justitie in Brouillard - zich op de OVVP konden beroepen tegenover hun lidstaat van oorsprong wat betreft een eventuele weigering tot toelating vanwege de 'openbaar gezag'-uitzondering.

Bovendien is in dit deel opgemerkt dat de Federaal Administratieve Rechtbank niet heeft verduidelijkt of auditors überhaupt onder het secundaire of primaire recht onder het acquis suisse vallen. Het is naar de mening van auteur dezes niet overtuigend dat zij niet zouden kunnen profiteren van vrij verkeer, omdat dit beroep niet wordt genoemd in de uitzonderingen met betrekking tot de reikwijdte van de OVVP.

Tot slot werd de positie van de juristen die nog geen beroepskwalificatie (e.g. titel van advocaat) hebben onderzocht. Er werd aangetoond dat, volgens de uitleg van de Zwitserse federale overheid en op basis van de jurisprudentie van het Hof van Justitie van de Europese Unie in de zaak Morgenbesser (en Peśla), een holistische visie moet worden gehanteerd, d.w.z. een holistische kijk op het kennisniveau van stagiairs bij de erkenning van diploma's en de toelating tot een stage in Zwitserland. Dit standpunt wordt ook ondersteund door de jurisprudentie van het Bundesgericht. Het Bundesgericht heeft de Peśla-jurisprudentie in principe gevolgd in overeenstemming met een recente mijlpaalbeslissing van januari 2020, ook al leidde dit in casu niet tot toelating tot de beroepsopleiding. 


\section{Zusammenfassung}

Diese Studie zeigt die gegenseitige Anerkennung von beruflichen Qualifikationen, in anderen Worten die Diplomanerkennung, zwischen der Schweiz und der EU, namentlich für ausgewählte Gesundheits- und Rechtsberufe. Diese Arbeit behandelt nicht nur das anwendbare Sekundärrecht, insbesondere die Richtlinie 2005/36/EG ('Berufsanerkennungsrichtlinie'), sondern sie untersucht auch, ob Primärrecht zwischen der Schweiz und der EU Anwendung findet. Für diesen Zweck wurde der institutionelle Rahmen, welcher mit diesem Thema eng verknüpft ist, dargestellt. Die zukünftigen Beziehungen zwischen der Schweiz und der EU, namentlich das Rahmenabkommen, könnten nämlich die zukünftige Auslegung des acquis suisse massgeblich beeinflussen.

\subsection{Institutioneller Rahmen zwischen der Schweiz und der EU (Teil I)}

In Teil I behandelt diese Untersuchung den institutionellen Rahmen zwischen der Schweiz und der EU und wie dieser in Zukunft geändert werden soll. Es werden im ersten Teil die Stolpersteine aufgezeigt, die bisher den sogenannten bilateralen Weg nicht gestoppt haben, namentlich die Masseneinwanderungsinitiative. Dies beinhaltet auch die momentanen Herausforderungen zur Suche eines geeigneten Gremiums für die einheitliche Auslegung der bilateralen Abkommen.

Im Frühling 2019 war der Entwurf für ein Rahmenabkommen zwischen der Schweiz und der EU in der öffentlichen Vernehmlassung. Dieses Rahmenabkommen würde ein Schiedsgericht schaffen, das es ermöglichen würde, dem Europäischen Gerichtshof (EuGH) für bestimmte Marktzugangsabkommen Fragen vorzulegen. Bei genauerer Betrachtung zeigt sich, dass die Einflussnahme des EuGH für die Auslegung dieser Marktzugangsabkommen relativ moderat ausfällt. Dabei wäre diese Form der Streitschlichtung wohl auch im Einklang mit der Autonomie des Unionsrechts. An dieser Stelle wurde auch im Vergleich mit anderen Streitschlichtungsmechanismen andiskutiert, ob das Schiedsgericht einen Ermessensspielraum besitzt, um Fragen dem Europäischen Gerichtshof vorzulegen, was bejaht werden kann (Anwendung der acte clair-Doktrin).

Der Entwurf für das Rahmenabkommen stellt einen Kompromiss zwischen Anliegen der Schweiz und der EU dar, welcher für viele Schweizer schwierig zu akzeptieren sein wird, da er bestimmte rote Linien überschreitet, insbesondere die 8-Tage-Regelung für 
Dienstleistungserbringer (ohne ein klassische Schere zwischen Politikern des linken und rechten Flügels). Zusätzlich zur Überschreitung der roten Linien gibt es den Einwand, dass es sich beim EuGH, um ein Gericht der Gegenseite handelt, womit unterstellt wird, dass dieser nicht unparteiisch entscheidet.

Zudem wurde die Auslegung von internationalen Verträgen und insbesondere die Auslegung des Freizügigkeitsabkommens zwischen der Schweiz und der EU (FZA) erforscht. Während der Europäische Gerichtshof (EuGH) im Ankara-Abkommen in vielen Fällen ähnlich progressiv wie im Binnenmarktrecht entschieden hat, wurde darauf hingewiesen, dass der EuGH im Ankara-Abkommen im Fall Demirkan und auch unter dem FZA im Fall Grimme zum Polydorprinzip zurückgekehrt ist. So wurde etwa von einem Generalanwalt im Fall Ettwein sogar angezweifelt, ob Primärrecht Teil des FZA sei, oder ob nur das ausdrücklich erwähnte Sekundärrecht unter dem acquis suisse Anwendung finde. Es wurde an dieser Stelle auch darauf hingewiesen, dass nach einigen Autoren die Rückkehr zum Polydorprinzip auf einige restriktive Urteile von nationalen Gerichten zurückzuführen ist. Angesichts der kürzlich ergangenen restriktiven Urteile der strafrechtlichen Abteilung des Schweizer Bundesgerichts ist es durchaus vorstellbar, dass das Polydorprinzip in der Rechtsprechung des EuGH vermehrt zum Tragen kommen wird, da die Auslegung massgeblich vom Kontext des jeweiligen Abkommens abhängt. Trotz der Rückkehr zum Polydorprinzip unter dem FZA wurde gezeigt, dass das Bundesgericht dem EuGH folgt, auch wenn letzterer seine Rechtsprechung ändert. Der EuGH dagegen ist dem Bundesgericht mit einer Ausnahme noch nie ausdrücklich gefolgt. Dabei wurde auch gezeigt, dass die Zusammenarbeit zwischen den Gerichten bzw. der richterliche Dialog, wie er zwischen dem EuGH und dem EFTA-Gerichtshof zu beobachten ist, zwischen dem Bundesgericht und dem EuGH nicht stattfindet. Der richterliche Dialog ist ausdrücklich im Rahmenabkommen enthalten. Es wurde dabei erklärt, dass der EFTA-Gerichtshof über diesen richterlichen Dialog hinausgeht und ein umgekehrtes Polydorprinzip entwickelt hat. Dieses hat zur Folge, dass der EFTA-Gerichtshof auch eine andere Auslegung einer Gesetzesbestimmung als der EuGH befürwortet, sofern das gleiche Resultat erreicht wird wie im Binnenmarktrecht.

Klar erscheint, dass eine Auslegungsregel nur für diejenigen Konzepte Geltung erlangen kann, welche überhaupt Bestandteil des FZA bilden. Es ist indessen nicht gelöst, wie die Konzepte bestimmt werden, die Bestandteil des acquis suisse sind. Die komplexe Struktur des FZA und die Kombination aus primär- sowie sekundärrechtlichen Bestimmungen macht 
es jedenfalls nicht leicht, um die anwendbaren Konzepte herauszuschälen. Diese Problematik wird auch durch das Rahmenabkommen nicht entschärft.

\subsection{Personenfreizügigkeit zwischen der Schweiz und der EU (Teil II)}

Teil II diskutiert die Grundfreiheiten, welche im acquis suisse nur teilweise verwirklicht sind. Während die direkte als auch die indirekte Diskriminierung für alle anwendbaren Grundfreiheiten (unter dem Vorbehalt der Rechtfertigung) verboten sind (Arbeitnehmer, Niederlassung und Dienstleistungen) und Drittwirkung zeitigen (kürzlich wurde letzteres höchstrichterlich auch offengelassen), ist es immer noch umstritten, ob das FZA ein Beschränkungsverbot enthält. Es gibt zurzeit keine Entscheidung des EuGH, die ein Beschränkungsverbot für das FZA (oder für ein anderes Assoziierungsabkommen) ausdrücklich bejahen würde. ${ }^{2431}$ Das Bundesgericht hat sich nicht offen dazu geäussert, aber implizit sich dafür ausgesprochen, indem es die primärrechtliche Anerkennung von Diplomen (basierend auf einem eher pragmatischen Ansatz bzw. wohl gestützt auf das Verhältnismässigkeitsprinzip) bejaht hat.

Während sich der EuGH im kürzlich ergangenen Urteil Picart (Grosse Kammer) das FZA streng nach seinem Wortlaut ausgelegt hat, wonach ein Staatsangehöriger der Schweiz oder der EU als Selbständiger Wohnsitz im Hoheitsgebiet der anderen Vertragspartei haben muss, hat der EuGH in der Rechtssache Wächtler (Grosse Kammer) festgehalten, dass auch Behinderungen der Freizügigkeit verboten sind, die vom betroffenen Herkunftsstaat ausgehen. Für die Diplomanerkennung haben die Schweizer Gerichte in weitgehend konstanter Rechtsprechung und im Einklang mit dem Binnenmarktrecht entschieden, dass ein grenzüberschreitender Bezug für die Anwendung des FZA und der Berufsanerkennungsrichtlinie genüge.

Im Übrigen wurde auch erwähnt, dass Personen, die keine Erwerbstätigkeit ausüben (z.B. Studenten) wegen dem Wortlaut von Artikel 24 Absatz 4 von Anhang I FZA insbesondere für den Zugang zur Hochschulbildung wohl nicht in den Geltungsbereich des FZA fallen. Selbst wenn Personen, die keine Erwerbstätigkeit ausüben, sich in dieser Situation nach der

2431 Der Begriff 'Beschränkung' wurde durch den EuGH benutzt: siehe Case C-581/17, Martin Wächtler v Finanzamt Konstanz, ECLI:EU:C:2019:138, para. 67. Obschon der EuGH den Begriff 'Beschränkung' benutzte, kann - nach dem in dieser Studie zum Polydorprinzip Gesagten - aus demselben Wortlaut nicht automatisch dieselbe Bedeutung eines Begriffs wie im Binnenmarktrecht abgeleitet werden. 
Rechtsprechung auch nicht auf Artikel 2 FZA (Nichtdiskriminierung analog zu Artikel 18 AEUV und entgegen den Entscheiden unterer Gerichte) berufen können, so können sich etwa wirtschaftlich aktive Studenten grundsätzlich auf die Grundfreiheiten in Verbindung mit Artikel 2 FZA berufen.

\subsection{Anerkennung der beruflichen Qualifikationen (Diplomanerkennung; Teil III)}

Teil III erhellt die Entstehung der grundlegenden Prinzipien der Diplomanerkennung. Die Entwicklung der Prinzipien des gegenseitigen Vertrauens und des Herkunftslandsprinzip beruhen auf der Rechtsprechung zu den Grundfreiheiten. Es wurde anhand der einschlägigen Rechtsprechung erneut hervorgehoben, dass namentlich das Schweizer Bundesgericht implizit die primärrechtliche Anerkennung von Diplomen basierend auf einem pragmatischen Ansatz (wohl gestützt auf den Verhältnismässigkeitsgrundsatz) befürwortet, während die Schweizer Bundesverwaltung sich sogar offen für die primärrechtliche Anerkennung gestützt auf die Rechtsprechung in Sachen Hocsman und Morgenbesser ausgesprochen hat. Die Erörterung der Rechtsprechung zum Beruf des Elektrikers zeigt, dass auch das Bundesverwaltungsgericht implizit diesem Ansatz folgen könnte, da die sekundärrechtliche Regelung zur Teilanerkennung zurzeit noch nicht Bestandteil des acquis suisse ist. Es wurde indessen gezeigt, dass dogmatisch betrachtet eine primärrechtliche Anerkennung nicht nur auf den Verhältnismässigkeitsgrundsatz, sondern entweder auf das Beschränkungsverbot oder aber auf einen rein pragmatischen Ansatz gestützt werden kann (ähnlich dem umgekehrten Polydorprinzip des EFTA-Gerichtshofes). Diese Auseinandersetzung hätte Einfluss auf die (primärrechtliche Anerkennung) von Drittstaatsdiplomen sowie die (primärrechtliche) Teilanerkennung von Diplomen. Dies widerspricht früherer und wenig bekannter Rechtsprechung des Bundesverwaltungsgerichts, welche die (primärrechtliche) Anerkennung gemäss der Rechtsprechung in Vlassopoulou und Dressen ausdrücklich ablehnte.

In diesem Zusammenhang wurde auch die Rechtsprechung in der Sache Tennah-Durez untersucht, in welcher der EuGH entschieden hat, dass Mitgliedsstaaten unter dem sektoriellen System auch Diplome anerkennen müssen, welche nicht auf einer überwiegend in der Gemeinschaft absolvierten Berufsausbildung beruhten, solange das Diplom von einem 
EU-Mitgliedsstaat ausgestellt ist, wobei nach Auffassung der Kommission diese Rechtsprechung auch unter der aktuellen Berufsanerkennungsrichtlinie einschlägig ist.

Mit Blick auf den Austritt des Vereinigten Königreichs aus der EU wurde der Schutz von erworbenen Rechten sowie Anwartschaften erörtert. Einige der rechtlichen Unklarheiten konnten durch den Entwurf für ein Abkommen zwischen der Schweiz und Grossbritannien über die Rechte der Bürgerinnen und Bürger beim Wegfall des Freizügigkeitsabkommens geklärt werden. Dieses schützt in Bezug auf die Diplomanerkennung erworbene Rechte und auch Anwartschaften und sogar bestimmte Personen, die noch nicht über ein Diplom verfügen. Ebenso ist die Anerkennung von Drittstaatsdiplomen geregelt. Nicht im Abkommen enthalten sind Regelungen über die primärrechtliche Anerkennung. Schliesslich fehlt in diesem Abkommen die Anerkennung für den Beruf des Revisionsexperten (Ausbildung als Wirtschaftsprüfer, Treuhandexperte oder Steuerexperte).

\subsection{Anerkennung der beruflichen Qualifikationen für ausgewählte Gesundheits- und Rechtsberufe (Teil IV)}

In diesem vierten Teil der Dissertation wurde auch grosses Gewicht auf die Auseinandersetzung mit der einschlägigen Rechtsprechung des EuGH sowie des EFTAGerichtshofes insbesondere für ausgewählte Gesundheits- und Rechtsberufe gelegt. Für die Berufsausübung einer Ärztin wurde eine interessante Entscheidung des EFTA-Gerichtshofes kommentiert, welche es dem Aufnahmemitgliedsstaat erlaubt, die Diplomanerkennung bei mangelnden Sprachkenntnissen zu verweigern. Nach der hier vertretenen Ansicht wäre es überzeugender, einen zweistufigen Ansatz zu wählen, welcher im Einklang mit der EuGHRechtsprechung steht und so auch von der Kommission in diesem Verfahren vorgebracht wurde. Mit dem zweistufigen Ansatz ist die Anerkennung von Diplomen unter dem sektoralen System automatisch, während die Berufsausübung mangels genügender Sprachkenntnisse verweigert werden kann.

Bei der Rechtsprechung des Bundesverwaltungsgerichts wurde auf einen sehr bedeutsamen Entscheid in Fünferbesetzung von 2017 hingewiesen, der kürzlich im Juni 2019 vom Bundesgericht aufgehoben wurde. Das Bundesverwaltungsgericht hatte zum ersten Mal entschieden, dass auch privatrechtliche Weiterbildungstitel für Ärzte ('Weiterbildung für den Schwerpunkt Reproduktionsmedizin und gynäkologische Endokrinologie') den Regeln der Diplomanerkennung unterliegen. Da die Spezialisierung nicht im Anhang V der 
Berufsanerkennungsrichtlinie aufgeführt ist, kam subsidiär Artikel 10 der

Berufsanerkennungsrichtlinie dafür zur Anwendung. Dabei hat das

Bundesverwaltungsgericht allerdings nicht diskutiert, ob besondere und aussergewöhnliche Gründe vorliegen, obschon die Rechtsprechung des EuGH in Angerer dies auch für die Anerkennung von Spezialisierungen verlangt, wobei dies konkret zum gleichen Ergebnis, nämlich zur Anwendbarkeit des allgemeinen Systems, führen würde. Schliesslich erwähnte das Bundesverwaltungsgericht mit Verweis auf den Entscheid des EuGH in Bobadilla obiter dictum, dass die Regeln der Diplomanerkennung auch bei nicht reglementierten Berufen zur Anwendung gelangen würden, wobei diese Aussage dogmatisch nicht näher erläutert wurde.

In einem wichtigen Leitentscheid des Bundesverwaltungsgerichtes von 2018 wurde eine Regelung, welche besagt, dass Ärzte drei Jahre praktische Erfahrung in der Schweiz benötigen als indirekt diskriminierend aber als gerechtfertigt eingestuft. Die Abteilung III urteilte, dass der Wortlaut von Artikel 55 der Berufsanerkennungsrichtlinie ${ }^{2432}$ zu wenig klar sei, um im Lichte der Rechtsprechung des Bundesgerichts zu Beschränkungen angewandt zu werden, obschon das Gericht die Massnahme als indirekt diskriminierend beurteilte. Die Standstill-Klausel wurde nicht geprüft, obwohl entgegen des zitierten Bundesgerichtsentscheids eine indirekte Diskriminierung vorlag. Es ist ungeklärt, ob Verletzungen der Standstill-Klausel in Analogie zur Rechtsprechung des EuGH unter dem Ankara-Abkommen gerechtfertigt werden können.

$\mathrm{Zu}$ den Gesundheitsberufen wurde die neue Regelung für Apotheker kurz behandelt, welche einen Weiterbildungstitel für Apotheker in eigener fachlicher Verantwortung verlangt. Gemäss der Rechtsprechung des EuGH fällt eine solche Spezialisierung unter das allgemeine System der Diplomanerkennung. Ungeachtet der Frage, ob diese Regelung entweder im Einklang mit den Vorgaben der Berufsanerkennungsrichtlinie steht oder aber nicht darunterfällt, weil nur die Ausübung des Berufes reglementiert wird, handelt es sich entweder um eine indirekte Diskriminierung oder um eine Beschränkung, welche gerechtfertigt sein muss (sofern Beschränkungen überhaupt unter das FZA fallen). Dies wird durch die einschlägige Rechtsprechung des EFTA-Gerichtshofes gestützt.

2432 'Unbeschadet des Artikels 5 Absatz 1 und des Artikels 6 Absatz 1 Buchstabe b gilt Folgendes: Mitgliedstaaten, die den Personen, die ihre Berufsqualifikationen in ihrem Hoheitsgebiet erworben haben, nur dann eine Kassenzulassung erteilen, wenn sie einen Vorbereitungslehrgang absolviert und/oder Berufserfahrung erworben haben, befreien die Personen, die ihre Berufsqualifikationen als Arzt bzw. Zahnarzt in einem anderen Mitgliedstaat erworben haben, von dieser Pflicht.' 
Während die schweizerische Umsetzung zu den Berufen des Anwalts und des Patentanwaltes sich mit Ausnahme eines Urteils, das Familienangehörigen von EU-Bürgern die Anrufung der Niederlassungsrichtlinie für Rechtsanwälte verweigerte, als weitgehend unproblematisch erweist, ist die rechtliche Situation für den Beruf des Notars momentan unbefriedigend, da die Gerichte diesbezüglich noch nicht für Klarstellung sorgen konnten.

Nach Ansicht der Wettbewerbskommission ist unter dem acquis suisse - aufgrund der Umsetzung von Titel II der Berufsanerkennungsrichtlinie im nationalen Recht und der Anwendung der Richtlinie vor der Revision - noch das Sekundärrecht für die Notare anwendbar. Es wurde festgestellt, dass subsidiär jedenfalls das Primärrecht für die Notare anwendbar ist. Somit ist die Freizügigkeit für die Notare auch unter dem acquis suisse zu gewähren. Für den Beruf des Notars wurde auch festgestellt, dass die Bereichsausnahme zur Ausübung hoheitlicher Gewalt im Lichte der spezifischen Auslegungsregel von Artikel 16 Absatz 2 FZA verstanden werden muss, was bedeutet, dass geringfügige sprachliche Differenzen entgegen vereinzelter Literaturmeinungen nicht zu einer vom Binnenmarktrecht abweichenden Auslegung der Bereichsausnahme führen können. Mit Blick auf die Bereichsausnahme wurde erforscht, dass angesichts der Rechtsprechung des EuGH in Brouillard diese Bereichsausnahme Schweizer Staatsangehörigen und Staatsangehörigen von Mitgliedsstaaten der EU von ihrem Herkunftsstaat nicht vorgehalten werden kann, wobei diesem Umstand in der Literatur bisher wenig Beachtung geschenkt wurde.

Für den Beruf des Revisors bzw. des Revisionsexperten (Ausbildung als Wirtschaftsprüfer, Treuhandexperte oder Steuerexperte) wurde auch für den Anwendungsbereich des FZA untersucht, dass bis heute unklar ist, ob dieser Beruf im Geltungsbereich des acquis suisse überhaupt unter das sekundärrechtliche oder das primärrechtliche Anerkennungsregime fällt. Schliesslich wurde auch darauf hingewiesen, dass es nicht überzeugt, zu behaupten, der Beruf des Revisionsexperten falle nicht in den Anwendungsbereich des FZA, da dieser sonst wohl in den Ausnahmebestimmungen aufgeführt wäre.

Letztlich wurde die Stellung von Rechtspraktikanten beleuchtet. Es wurde gezeigt, dass gemäss den Erläuterungen der schweizerischen Bundesverwaltung und gestützt auf die Rechtsprechung des EuGH im Fall Morgenbesser (und Peśla) eine holistische, d.h. eine gesamtheitliche Betrachtung, des Kenntnisstandes von Praktikanten bei der Diplomanerkennung und Zulassung zum Praktikum in der Schweiz erfolgen muss. Diese Ansicht wird auch durch die Rechtsprechung des Bundesgerichts gestützt. Das Bundesgericht 
Chapter 11

ist der Rechtsprechung in Sachen Peśla gemäss einem kürzlich ergangenen Leitentscheid vom Januar 2020 im Grundsatz gefolgt, auch wenn es den konkreten Fall abschlägig beurteilte.

$-452-$ 


\section{Résumé}

Cette étude présente la reconnaissance mutuelle des qualifications professionnelles, c'està-dire la reconnaissance des diplômes, entre la Suisse et l'UE, en particulier pour certaines professions du domaine de la santé et du droit. Cette étude ne traite pas seulement du droit secondaire applicable, en particulier la Directive 2005/36/CE ('Directive relative à la reconnaissance professionnelle'), mais examine également si le droit primaire est applicable entre la Suisse et l'UE. À cette fin, le cadre institutionnel, qui est étroitement lié à ce sujet, a été présenté. Les futures relations entre la Suisse et l'UE, en particulier l'accord-cadre, pourraient en effet avoir une influence significative sur l'interprétation future de l'acquis suisse.

\subsection{Cadre institutionnel entre la Suisse et l'UE (Partie I)}

La première partie de cette étude porte sur le cadre institutionnel entre la Suisse et l'UE et sur la manière dont celui-ci doit être modifié dans l'avenir. Dans la première partie, on identifie les pierres d'achoppement, qui n'ont pas encore arrêté la voie dite bilatérale, à savoir l'initiative d'immigration de masse. Cela inclut également les défis actuels pour trouver un organe approprié pour l'interprétation uniforme des accords bilatéraux.

Au printemps 2019, la consultation publique pour le projet d'accord-cadre entre la Suisse et l'UE a été lancée. Cet accord-cadre créerait un tribunal d'arbitrage qui permettrait de soumettre des questions à la Cour de justice de l'Union Européenne (CJUE) pour certains accords d'accès au marché. En y regardant de plus près, il apparaît clairement que l'influence de la CJUE sur l'interprétation de ces accords d'accès au marché est relativement modérée. En même temps, cette forme de règlement des différends serait probablement aussi compatible avec l'autonomie du droit de l'Union. À ce stade, il a également été discuté, en comparaison avec d'autres mécanismes de règlement des différends, si le tribunal arbitral dispose d'un pouvoir discrétionnaire pour soumettre des questions à la Cour de Justice de l'Union Européenne, ce qui peut être affirmé (application de la doctrine de l'acte clair).

Le projet d'accord-cadre représente un compromis entre les préoccupations de la Suisse et de l'UE, qui sera difficile à accepter pour de nombreux Suisses car il franchit certaines lignes rouges, notamment la règle des 8 jours pour les prestataires de services (sans les clivages classiques entre les politiciens de gauche et de droite). En plus de franchir les lignes 
rouges, il y a l'objection selon laquelle la CJUE est un tribunal de l'autre partie, ce qui implique qu'elle ne juge pas de manière impartiale.

En outre, l'interprétation des traités internationaux et en particulier l'interprétation de l'Accord sur la Libre Circulation des Personnes entre la Suisse et l'UE (ALCP) a été étudiée. Alors que la Cour de Justice de l'Union Européenne (CJUE) dans l'accord d'Ankara a, dans de nombreux cas, rendu des décisions tout aussi progressistes que dans le droit du marché intérieur, il a été souligné que la CJUE est revenue au Polydorprinzip (i.e. la jurisprudence de Polydor) dans l'affaire Demirkan (dans le contexte de l'accord d'Ankara) et également dans l'affaire Grimme (dans le contexte de l'ALCP).

Ainsi, dans l'affaire Ettwein, un Avocat-Général s'est même demandé si le droit primaire faisait partie de l'ALCP ou si seul le droit secondaire expressément mentionné était applicable en vertu de l'acquis suisse. A ce stade, il a également été souligné que, selon certains auteurs, le retour au Polydorprinzip est dû à certains jugements restrictifs des tribunaux nationaux. $\mathrm{Au}$ vu des récents arrêts restrictifs de la division du droit pénal du Tribunal Fédéral Suisse, il est tout à fait concevable que le Polydorprinzip soit de plus en plus appliqué dans la jurisprudence de la CJUE, puisque l'interprétation dépend largement du contexte de l'accord respectif. Malgré le retour au Polydorprinzip dans le cadre de l'ALCP, il a été démontré que le Tribunal Fédéral suit la CJUE, même si cette dernière modifie sa jurisprudence. En revanche, la CJUE n'a jamais explicitement suivi le Tribunal Fédéral, à une exception près. Il a également été démontré que la coopération entre les tribunaux ou le dialogue judiciaire, comme on peut l'observer entre la CJUE et la Cour AELE, n'a pas lieu entre le Tribunal Fédéral et la CJUE. Cependant, le dialogue judiciaire est explicitement inclus dans l'accordcadre. Il a été constaté que la Cour de l'AELE va au-delà de ce dialogue judiciaire et a développé un Polydorprinzip inverse. En conséquence, la Cour de l'AELE soutient également une interprétation d'une disposition juridique différente de celle de la CJUE, à condition que le même résultat soit obtenu que dans le droit du marché intérieur.

Il semble évident qu'une règle d'interprétation ne peut s'appliquer qu'aux concepts qui font effectivement partie de l'ALCP. Toutefois, la question de savoir comment déterminer les concepts qui font partie de l'acquis suisse n'a pas été résolue. En tout état de cause, la structure complexe de l'ALCP et la combinaison de dispositions de droit primaire et de droit secondaire ne permettent pas de facilement identifier les concepts applicables. L'accord-cadre n'atténue pas non plus ce problème. 


\subsection{Libre circulation des personnes entre la Suisse et I'UE (Partie II)}

La deuxième partie traite des libertés fondamentales, qui ne sont que partiellement mises en œuvre dans l'acquis suisse. Si les discriminations directes et indirectes (sous réserve de justification) sont interdites pour toutes les libertés fondamentales applicables (travailleurs, établissement et services) et ont des effets à l'égard des tiers (ce dernier point a récemment été laissé en suspens par la plus haute juridiction), il est toujours contesté que l'ALCP contienne une interdiction de restrictions. Il n'existe actuellement aucune décision de la CJUE qui affirmerait explicitement une interdiction de restrictions pour l'ALCP (ou pour tout autre accord d'association). ${ }^{2433}$ Le Tribunal Fédéral ne s'est pas ouvertement prononcé sur ce point, mais a implicitement exprimé son soutien en affirmant la reconnaissance des diplômes en droit primaire selon une approche plutôt pragmatique (probablement fondée sur le principe de proportionnalité).

Alors que dans le récent arrêt Picart (Grande Chambre), la CJUE a interprété l'ALCP strictement en fonction de sa formulation, selon laquelle un ressortissant de la Suisse ou de l'UE doit résider en tant qu'indépendant sur le territoire de l'autre partie contractante, la CJUE dans l'arrêt Wächtler (Grande Chambre) a jugé que les obstacles à la libre circulation des personnes émanant de l'État d'origine concerné sont interdits. En ce qui concerne la reconnaissance des diplômes, les tribunaux suisses ont décidé, dans une jurisprudence largement cohérente et conformément au droit du marché intérieur, qu'un aspect transfrontalier est suffisant pour l'application de l'ALCP et de la Directive relative à la reconnaissance professionnelle.

En outre, il a également été mentionné que les personnes n'exerçant pas d'activité économique (par exemple les étudiants) sont susceptibles de ne pas relever du champ d'application de l'ALCP en raison du libellé de l'article 24, paragraphe 4, de l'annexe I de l'ALCP, notamment pour l'accès à l'enseignement supérieur. Donc, les personnes n'exerçant pas d'activité économique ne peuvent pas, selon la jurisprudence, invoquer l'article 2 de l'ALCP (non-discrimination analogue à l'article 18 du TFUE ; et contrairement aux décisions

2433 Le terme "restriction" a été utilisé par la CJUE : voir l'affaire C-581/17, Martin Wächtler contre Finanzamt Konstanz, ECLI:EU:C:2019:138, paragraphe 67. Bien que la CJUE ait utilisé le terme "restriction", la même formulation ne peut - selon ce qui a été dit dans cette étude sur le Polydorprinzip - donner automatiquement la même signification à un terme que dans le droit du marché intérieur. 
des juridictions inférieures). Cependant, les étudiants économiquement actifs, par exemple, peuvent en principe invoquer les libertés fondamentales en rapport avec l'article 2 de l'ALCP.

\subsection{Reconnaissance des qualifications professionnelles (reconnaissance des diplômes ; partie III)}

La troisième partie met en lumière l'émergence des principes de base de la reconnaissance des diplômes. Le développement des principes de confiance mutuelle et du principe du pays d'origine est basé sur la jurisprudence relative aux libertés fondamentales. Il a été rappelé, sur la base de la jurisprudence pertinente, que le Tribunal Fédéral soutient implicitement la reconnaissance des diplômes au titre du droit primaire sur la base d'une approche pragmatique (probablement fondée sur le principe de proportionnalité), tandis que l'administration fédérale suisse s'est même ouvertement déclarée en faveur de la reconnaissance au titre du droit primaire sur la base de la jurisprudence dans les affaires Hocsman et Morgenbesser. La discussion de la jurisprudence relative à la profession d'électricien montre que le Tribunal Administratif Fédéral pourrait également suivre implicitement cette approche, car le droit secondaire sur la reconnaissance partielle ne fait actuellement pas encore partie de l'acquis suisse. Toutefois, il a été démontré que, d'un point de vue dogmatique, la reconnaissance au titre du droit primaire peut être fondée non seulement sur le principe de proportionnalité, mais aussi soit sur l'interdiction des restrictions, soit sur une approche purement pragmatique (semblable au Polydorprinzip inverse de la Cour de l'AELE). Ce différend aurait un impact sur la reconnaissance (de droit primaire) des diplômes de pays tiers et la reconnaissance partielle (de droit primaire) des diplômes. Cela contredit la jurisprudence antérieure et peu connue du Tribunal Administratif Fédéral, qui a explicitement rejeté la reconnaissance (primaire) conformément à la jurisprudence dans l'affaire Vlassopoulou et Dressen.

Dans ce contexte, la jurisprudence dans l'affaire Tennah-Durez a également été examinée, dans laquelle la CJUE a jugé que les États membres doivent également reconnaître dans le cadre du système sectoriel les diplômes qui ne sont pas fondés sur une formation professionnelle acquise de manière prépondérante dans l'Union, pour autant que le diplôme soit délivré par un État membre de l'UE. La Commission considère que cette jurisprudence est également pertinente dans le cadre de la Directive actuelle relative aux qualifications professionnelles. 
La protection des droits acquis a été discutée dans la perspective du retrait du RoyaumeUni de l'UE. Certaines des ambiguïtés juridiques ont été clarifiées par le projet d'accord entre la Suisse et le Royaume-Uni sur les droits des citoyens en cas de retrait de l'Accord sur la Libre Circulation des Personnes (ALCP). Cet accord protège les droits acquis en matière de reconnaissance des diplômes, ainsi que les droits acquis et voire même certaines personnes qui n'ont pas encore de diplôme. La reconnaissance des diplômes de pays tiers est également réglementée. L'accord ne contient pas de dispositions sur la reconnaissance en vertu du droit primaire. Enfin, l'accord ne reconnaît pas la profession d'expert en audit (formation d'auditeur, d'expert fiduciaire ou d'expert fiscal).

\subsection{Reconnaissance des qualifications professionnelles pour certaines professions du domaine de la santé et du droit (Partie IV)}

Dans cette quatrième partie de la thèse, l'accent a été mis sur l'examen de la jurisprudence pertinente de la Cour de Justice de l'Union Européenne (CJUE) ainsi que de la Cour de l'AELE, en particulier pour certaines professions de la santé et du droit. Une décision intéressante de la Cour de l'AELE a été commentée concernant la pratique professionnelle d'une femme-médecin, qui permet à l'État membre d'accueil de refuser la reconnaissance du diplôme en cas de compétences linguistiques insuffisantes. Selon le point de vue exprimé ici, il serait plus convaincant d'adopter une approche en deux étapes, qui est conforme à la jurisprudence de la CJUE et a donc également été proposée par la Commission dans cette procédure. Avec l'approche en deux étapes, la reconnaissance des diplômes dans le cadre du système sectoriel est automatique, tandis que la pratique professionnelle peut être refusée en raison de compétences linguistiques insuffisantes.

Dans la jurisprudence du Tribunal Administratif Fédéral, il est fait référence à une décision très importante prise en 2017 par une formation de cinq membres, qui a été annulée par le Tribunal Fédéral en juin 2019. Le Tribunal Administratif Fédéral avait jugé pour la première fois que les titres de formation continue des médecins de droit privé ('formation continue pour la spécialisation en médecine de la reproduction et en endocrinologie gynécologique') étaient également soumis aux règles de reconnaissance des diplômes. Comme la spécialisation ne figure pas dans l'annexe $\mathrm{V}$ de la Directive relative à la reconnaissance des qualifications professionnelles, l'article 10 de la Directive a été appliqué de manière subsidiaire. Toutefois, le Tribunal Administratif Fédéral n'a pas examiné la 
question de savoir s'il existe des motifs spéciaux et exceptionnels, bien que la jurisprudence de la Cour de justice de l'Union Européenne dans l'affaire Angerer l'exige également pour la reconnaissance des spécialisations, bien que cela conduirait précisément au même résultat, à savoir l'applicabilité du système général. Enfin, le Tribunal Administratif Fédéral, se référant à la décision de la CJUE dans l'obiter dictum de Bobadilla, a mentionné que les règles de reconnaissance des diplômes s'appliqueraient également aux professions non réglementées, bien qu'aucune motivation soit donnée d'un point de vue dogmatique.

Dans une décision importante du Tribunal Administratif Fédéral en 2018, un règlement qui stipule que les médecins doivent avoir trois ans d'expérience pratique en Suisse a été classé comme indirectement discriminatoire mais justifié. La Cour III a jugé que le libellé de l'article 55 de la Directive relative aux qualifications professionnelles ${ }^{2434}$ n'était pas suffisamment clair pour être appliqué à la lumière de la jurisprudence du Tribunal Fédéral en matière de restrictions, bien que le Tribunal ait estimé que la mesure était indirectement discriminatoire. La clause de standstill n'a pas été examinée, même si, contrairement à la décision citée du Tribunal Fédéral, il existe une discrimination indirecte. Il n'est pas clair si les violations de la clause de standstill peuvent être justifiées par analogie avec la jurisprudence de la CJUE dans le cadre de l'accord d'Ankara.

En ce qui concerne les professions du domaine de la santé, le nouveau règlement pour les pharmaciens, qui exige un titre de formation continue pour les pharmaciens indépendants, a été brièvement discuté. Selon la jurisprudence de la CJUE, cette spécialisation relève du système général de reconnaissance des diplômes. Indépendamment de la question de savoir si ce système est conforme ou non aux dispositions de la Directive relative à la reconnaissance des qualifications professionnelles, parce que seul l'exercice de la profession est réglementé, il constitue soit une discrimination indirecte, soit une restriction qui doit être justifiée (si les restrictions sont couvertes par l'ALCP). Ceci est étayé par la jurisprudence pertinente de la Cour de l'AELE.

Si la transposition suisse concernant les professions d'avocat et de conseil en brevets ne pose pas de problème majeur, à l'exception d'un arrêt qui a refusé aux membres de la famille

${ }^{2434}$ Sans préjudice de l'article 5, paragraphe 1, et de l'article 6, premier alinéa, point b), les États membres qui exigent des personnes ayant acquis leurs qualifications professionnelles sur leur territoire l'accomplissement d'un stage préparatoire et/ou une période d'expérience professionnelle pour être conventionnés d'une caisse d'assurance-maladie dispensent de cette obligation les titulaires des qualifications professionnelles de médecin et de praticien de l'art dentaire acquises dans un autre État membre. 
de citoyens de l'UE d'invoquer la Directive sur l'établissement pour les avocats, la situation juridique pour la profession de notaire est actuellement insatisfaisante, car les tribunaux n'ont pas encore pu fournir de précisions à cet égard.

Selon la commission de la concurrence, en vertu de l'acquis suisse - en raison de la transposition du titre II de la Directive relative à la reconnaissance professionnelle en droit national et de l'application de la Directive avant sa révision - le droit secondaire est toujours applicable aux notaires. Il a été établi que le droit primaire est en tout état de cause subsidiairement applicable aux notaires. Par conséquent, la libre circulation des notaires doit également être accordée en vertu de l'acquis suisse. Pour la profession de notaire, il a également été établi que l'exception pour l'exercice de l'autorité souveraine doit être comprise à la lumière de la règle d'interprétation spécifique de l'article 16, paragraphe 2, de l'ALCP, ce qui signifie que, contrairement aux opinions isolées dans la littérature, des différences linguistiques mineures ne peuvent pas conduire à une interprétation de l'exception qui s'écarte du droit du marché intérieur. En ce qui concerne l'exception sectorielle, les analyses ont montré que, compte tenu de la jurisprudence de la CJUE dans l'affaire Brouillard, cette exception sectorielle ne peut pas être opposée aux ressortissants suisses et aux ressortissants des États membres de l'UE par leur pays d'origine, bien que cette circonstance ait jusqu'ici reçu peu d'attention dans la littérature.

En ce qui concerne la profession de l'auditeur ou de l'expert réviseur (formation d'auditeur, d'expert fiduciaire ou d'expert fiscal), il a également été examiné pour le champ d'application de l'ALCP qu'il n'est toujours pas clair si cette profession, relevant du champ d'application de l'acquis suisse, relève du régime de reconnaissance du droit secondaire ou du droit primaire. Enfin, il a également été souligné qu'il n'est pas convaincant d'affirmer que la profession d'expert en audit ne relève pas du champ d'application de l'ALCP, car sinon elle serait probablement répertoriée dans les dispositions d'exemption.

Enfin, la situation des stagiaires du domaine du droit a été examinée. Il a été démontré que, selon les explications de l'administration fédérale suisse et sur la base de la jurisprudence de la Cour de Justice de l'Union Européenne dans l'affaire Morgenbesser (et Peśla), il faut adopter une vision globale, c'est-à-dire une vue d'ensemble du niveau de connaissances des stagiaires en matière de reconnaissance des diplômes et d'admission aux stages en Suisse. Cette opinion est également soutenue par la jurisprudence du Tribunal Fédéral. Le Tribunal Fédéral a en principe appliqué la jurisprudence sur Peśla conformément à une récente décision historique de janvier 2020, même si la décision a été négative ans le cas spécifique. 